\title{
THERMAL STRESS IN BONDED DISSMILAR ELASTIC HALF PLANES CONTAINING A GRIFFITH CRACK AT THE INTERFACE
}

\section{RAJENDRA KUMAR TRIPATHI ${ }^{\mathrm{a} 1}$, P.N. PATHAK ${ }^{\mathrm{b}}$ AND SHAVEJ ALI SIDDIQUI ${ }^{\mathrm{c}}$}

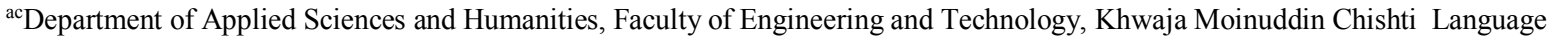 \\ University, Lucknow, U.P., India \\ ${ }^{b}$ Department of Mathematics, Axis Institute of Higher Education, C.S.J.M.U., Kanpur, U.P., India
}

\begin{abstract}
This paper concerns with the problem of determine the thermal stress distribution in bonded dissimilar elastic half planes containing a Griffith crack at the interface. The deformation in two half planes is due to the application of temperature to the faces of the crack. The analysis is carried out by a system of dual integral equation which are farther reduced to the solutions of Riemann Hilbert problem. An exact solution of these equations is obtained by using Fourier transforms and expressions for the quantities interest are obtained in closed form. The components of stress been calculated in the case of constant prescribed temperature.
\end{abstract}

KEYWORDS: Riemann Hilbert Problems, Fourier Transforms, Griffith Cracks, Heat Conduction, Modulus of Elasticity, Singular Integral Equations

In recent years, a few problems have attempted for calculating thermal stresses in dissimilar media (Brown and Erdogan, 1968) (Bregman and Kabsir, 1974). Here we investigate the problem of the distribution of thermal stress in bonded dissimilar elastic half planes containing a Griffith crack at the interface. The problem is first reduced to a system of simultaneous dual integral equations which are further reduced to two singular integral equations. The solution of these equations is obtained by the method given in (Lowengrub and Sneddon, 1973), These solutions have been used to determine components of stress. The calculations have been done in case when constant temperature is prescribed on crack surface. It has been shown that the stress components have singularity of the form $\frac{1}{\gamma\left(n^{2}-1\right)} \frac{\cos }{\sin }\left(\gamma \log \left|\frac{x+1}{x-1}\right|\right)$ at the rim of the crack.

\section{FORMULATION OF THE PROBLEM}

We assume that two semi-infinite media with thermoelastic properties $\propto_{j}, \bar{K}_{j, \mu_{j}} K_{j}(j=1,2)$, Where $\propto_{j}$ is the coefficient of thermal expansion, $\mathrm{Kj}$ is the coefficient of heat conduction $\mu_{j}$ is the modulus of rigidity and $\bar{K}=3-4 \eta_{j}, \eta_{j}$ being poisson's ratio of the elastic material. Suffixes $1 \& 2$ and correspond to the upper and lower half planes bonded together along the $\mathrm{x}$ aixs except over the region $|x| \leq 1, y=0$. For a symmertical deformation of the solid, the displacement vector may be taken to have components $\left[\mathrm{u}_{\mathrm{x}}(\mathrm{x}, \mathrm{y}), \mathrm{u}_{\mathrm{y}}(\mathrm{x}\right.$, y)] and the non-vanishing components of stress tensor are $\sigma_{x}, \sigma_{y}$ and $\sigma_{x y}$.

If we assume that the upper and lower faces of the crack are each subjected to a prescribed pressure $\mathrm{p}(\mathrm{x})$ and temperature $\mathrm{T}(\mathrm{x})$. We see that inside the crack area we have the conditions.

$\sigma_{y y}\left(x, 0^{+}\right)=\sigma_{y y}\left(x, 0^{-}\right)=-p(x),|x| \leq 1$

$\sigma_{x y}\left(x, 0^{+}\right)=\sigma_{x y}\left(x, 0^{-}\right)=-p(x),|x| \leq 1$

$T\left(x, 0^{+}\right)=T\left(x, 0^{-}\right)=-T(x),|x| \leq 1$

For the region of the interface not occupied by the crack, the following continuity conditions must be satisfied:

$u_{x}\left(x, 0^{+}\right)=u_{x}\left(x, 0^{-}\right)=|x|>1$

$u_{y}\left(x, 0^{-}\right)=u_{y}\left(x, 0^{-}\right)=|x|>1$

$\sigma_{x y}\left(x, 0^{+}\right)=\sigma_{x y}\left(x, 0^{-}\right)=|x|>1$

$\sigma_{y y}\left(x, 0^{+}\right)=\sigma_{y y}\left(x, 0^{-}\right)=|x|>1$

$T\left(x, 0^{+}\right)=T\left(x, 0^{-}\right)=|x|>1$

$\bar{K} \frac{\partial T}{\partial y}\left|y=0^{+}=\overline{K_{2} \frac{\partial T}{\partial y}}\right| y=0^{-}|x|>1$

As the solution of the equations of elastic equilibrium we take the displacement field 
$u_{x}(x, y)=$

$\frac{i F * \xi^{-1}\left(A_{1}-K_{1}^{-1}\left(A_{1}-B_{1}\right)|\xi|\left(y+\Omega_{1}\right) e|\xi| y ; \xi \rightarrow\right], y \geq 0}{i F * \xi^{-1}\left(A_{1}-\bar{K}_{1}^{-1}\left(A_{1}-B_{1}\right)|\xi|\left(y+\Omega_{1}\right) e|\xi| y ; \xi \rightarrow\right], y \geq 0}$

$u_{y}(x, y)=$

$\frac{i F * \xi^{-1}\left(B_{1}-K_{1}^{-1}\left(A_{1}-B_{1}\right)|\xi|\left(y+\Omega_{1}\right) e^{-|\xi| y} ; \xi \rightarrow x, y \geq 0\right.}{i F * \xi^{-1}\left(B_{1}-\bar{K}_{3}^{-1}\left(A_{2}-B_{2}\right) \mid \xi\left(y+\Omega_{2}\right) e^{-|\xi| y} x ; \xi \rightarrow x, y \geq 0\right.}$

$T_{x}(x, y)=\frac{\left.F * \frac{\left[\left(\Omega_{1}(\xi)\right]\right.}{\left(1+\eta_{1}\right) \alpha_{1}} e^{-|\xi| y}, \xi \rightarrow x\right], y \geq 0}{\left.F * \frac{\left[\left(\Omega_{2}(\xi)\right]\right.}{\left(1 \eta_{2}\right) \alpha_{2}} e^{-|\xi| y}, \xi \rightarrow x\right], y \leq 0}$

$\sigma_{x y}(x, y)=$

$\frac{i F *\left[-\mu_{1} e^{-|\xi| y}\left(K_{1}^{-1}\left[A_{1}\left(1+K_{1}\right)+B_{1}\left(K_{1}-1\right)-2\left(A_{1}-B_{1}\right)\right] \mid \xi \rightarrow x, y \geq 0\right.\right.}{i F *\left[-\mu_{1} e^{-|\xi| y}\left(K_{1}^{-1}\left[A_{2}\left(1+K_{2}\right)+B_{2}\left(K_{2}-1\right)+2\left(A_{2}-B_{2}\right)\right] \mid \xi \rightarrow x, y \leq 0\right.\right.}$

$\sigma_{y y}(x, y)=$ $\frac{i F *\left[-\mu_{1} e^{-|\xi| y}\left(K_{2}^{-1}\left[\left(1+K_{1}\right)+B_{1}\left(K_{1}-1\right)-2\left(A_{1}-B_{1}\right)\right]|\xi| y|,| \xi \rightarrow x \geq 0\right.\right.}{i F *\left[-\mu_{1} e^{-|\xi| y}\left(K_{2}^{-1}\left[B_{2}\left(1+K_{2}\right)+A_{2}\left(K_{2}-1\right)+2\left(A_{2}-B_{2}\right)\right]|\xi| y|,| \mid \xi \rightarrow x\right], y \leq 0\right.}$ (2.11)

In these equations denotes the operator defined by the equation

$F *[\emptyset(\xi, y), \xi \rightarrow x]=\frac{1}{\gamma(2 \pi)} \int_{-\infty}^{\infty} \phi(\xi, y) e^{-|\xi| y} d \xi$

On applying the conditions (2.3) and (2.6) we have

$\left(\Omega_{2}(\xi)\right)=\alpha_{2}\left(1+\eta_{2} / \alpha_{1}\left(1+\eta_{1}\right) \Omega_{2}(\xi)\right.$

and

$\left.\xi F *\left\{\frac{\bar{K}_{1} \Omega_{1}(\xi)}{\alpha_{1}\left(1+\eta_{2}\right)}+\frac{\overline{K_{2}} \Omega_{2}(\xi)}{\alpha_{2}\left(1+\eta_{2}\right)}\right\}, \xi \rightarrow x\right]=0$

or

$\left(\xi F * \Omega_{1}(\xi), \xi \rightarrow x\right)=0, x>1$.

Hence from condition $(2,3)$ we get the following pair of dual integral equations

$F * \Omega_{1}(\xi), \xi \rightarrow x=-\alpha_{1}\left(1+\eta_{1}\right) T(x), x<1$

$F *\left[\xi \Omega_{2}(\xi),\right], \xi \rightarrow x=0, x>1$

Taking

$\xi \Omega_{1}(\xi)=\int_{0}^{1} \psi(t) \cos (\xi t) d t$,

and it is easily shown that

$\psi(t)=-\frac{2 \alpha_{1}\left(1+\eta_{1}\right)}{\pi} \frac{d}{d t} \int_{0}^{1} \frac{x T(x)}{\sqrt{\left(t^{2}-x^{2}\right)}} d x$
From equations (2.1) and (2.5) we see that $\sigma_{y y}$ $\left(\mathrm{x}, 0^{+}\right)=\sigma_{y y}\left(\mathrm{x}, 0^{-}\right)$for al values of $\mathrm{X}$ and it is easily shown that this condition is equivalent to the equation

$$
\begin{aligned}
K_{1}\left(K_{2}-1\right) \Gamma A_{2}- & K_{2}\left(K_{2}+1\right) B_{2} \Gamma \\
& =K_{2}\left(K_{1}-1\right) A_{1}+K_{2}\left(K_{1}+1\right) B_{1}
\end{aligned}
$$

Where

$\Gamma=\mu_{2} / \mu_{1}$

Similarly we can show that the boundary conditions (2.2) and (2.5) are equivalent to the equation

$$
\begin{aligned}
K_{1}\left(K_{2}+1\right) A_{2} \Gamma- & K_{1}\left(K_{2}-1\right) B_{2} \Gamma \\
& =K_{2}\left(K_{1}+1\right) A_{1}-K_{2}\left(K_{1}-1\right) B_{1}
\end{aligned}
$$

Solving these equations for $A_{2}$ and $B_{2}$ in terms of $A_{1}$ and $B_{1}$ we have

$$
\begin{aligned}
& K_{1} \Gamma A_{2}(\xi)=\frac{1}{2}\left(K_{1} K_{2}+1\right) A_{1}(\xi)-\frac{1}{2}\left(K_{1} K_{2}-1\right) A_{1}(\xi) \\
& K_{1} \Gamma B_{2}(\xi)=\frac{1}{2}\left(K_{1} K_{2}-1\right) A_{1}(\xi)-\frac{1}{2}\left(K_{1} K_{2}+1\right) B_{1}(\xi)
\end{aligned}
$$

From which we deduce immediately that

$\Gamma\left(A_{1}+B_{2}\right)=-K_{2}\left(A_{1}+B_{1}\right)$.

Now from equations (2.7) and (2.8) we see that the boundary conditions (2.4) are equivalent to the conditions

$F *\left\{\frac{\xi^{-1}\left\{A_{1}(\xi)-A_{2}(\xi)+\Omega_{1}(\xi)-\Omega_{2}(\xi),=x|=| x \mid>1\right.}{\xi^{-1}\left\{B_{1}(\xi)-B_{2}(\xi)+\Omega_{1}(\xi)-\Omega_{2}(\xi),=x|=| x \mid>1\right.}\right\}$

Respectively

Now using (2.17) we may reduce these in turn to

$F * i \xi^{-1}\left\{\left[K_{1} \Gamma+\frac{1}{2}\left(K_{1} K_{2}+1\right) A_{1}(\xi)+\frac{1}{2}\left(K_{1} K_{2}-\right.\right.\right.$

1) $\left.\left.B_{2}(\xi)+K_{1} \Gamma\left(\Omega_{1}+\Omega_{2}\right)\right\}, x\right]=0,|x|>1$

$F * i|\xi|^{-1} \frac{1}{2}\left(K_{1} K_{2}-1\right) A_{1}(\xi)+\left(K_{1} \Gamma+\frac{1}{2}\right)\left(K_{1} K_{2}+\right.$

1)] $\left.\left.\left(B_{1}\right)(\Sigma)-K_{1} \Gamma\left(\Omega_{1}+\Omega_{2}\right)\right\}, x\right]=0,|x|>1$

And for $|\mathrm{x}|>1$, we get from (2.1) and (2.11)

$$
\begin{aligned}
& \begin{array}{l}
F *\left[\left(K_{1}-1\right) A_{1}\left(K_{1}+1\right)(\xi)+\left(K_{2}-1\right) B_{1}(\xi), x\right] \\
\quad=K_{1} \mu_{1}^{-1} p(x),|x|<1
\end{array} \\
& F *\left[i \operatorname{sgn} \xi\left\{\left(K_{1}+1\right) A_{1}(\xi)+\left(K_{2}-1\right) B_{1}(\xi), x\right]=\right. \\
& 0|x|<1
\end{aligned}
$$


On setting we have

$$
\begin{aligned}
& {\left[\left(K_{1} \Gamma+\frac{1}{2}\left(K_{1} K_{2}+1\right) A_{1}(\xi)+\frac{1}{2}\left(K_{1} K_{2}-1\right) B_{1}(\xi)\right.\right.} \\
& +K_{1} \Gamma\left(\Omega_{1}-\Omega_{2}\right)=M(\xi) \\
& \frac{1}{2}\left(K_{1} K_{2}-1\right) A_{1}(\xi)+\left(K_{1} \Gamma+\frac{1}{2}\right)\left(K_{1} K_{2}+1\right) B_{1}(\xi)- \\
& K_{1} \Gamma\left(\Omega_{1}(\xi)+\Omega_{2}(\xi)\right)=N(\xi)
\end{aligned}
$$

A Simple calculation shows that

$$
\begin{aligned}
& K_{1}\left(K_{2}+\Gamma\right)\left(1+K_{1} \Gamma\right) A_{1}(\xi)=\left(K_{1} \Gamma+\frac{1}{2}\right)\left(K_{1} K_{1}+\right. \\
& 1) M(\xi)-\frac{1}{2}\left(K_{1} K_{2}-1\right) N(\xi)- \\
& K_{1} \Gamma \Omega_{1}(\xi)\left[K_{1} \Gamma \frac{1}{2} K_{1} K_{2}\right]+K_{1} \Gamma \Omega_{2}(\xi) \times\left(K_{1} \Gamma+1\right)
\end{aligned}
$$

$K_{1}\left(K_{2}+\Gamma\right)\left(1+K_{1} \Gamma\right) B_{1}(\xi)=\left(K_{1} \Gamma+\frac{1}{2}\right)\left(K_{1} K_{2}+\right.$ 1) $M(\xi)-\frac{1}{2}\left(K_{1} K_{2}-1\right) N(\xi)-K_{1} \Gamma \Omega_{1}(\xi)\left[K_{1} \Gamma K_{1} K_{2}\right]+$ $K_{1} \Gamma \Omega_{2}(\xi) \times\left(K_{1} \Gamma+1\right)$

We reduce the equations (2.19) through (2.20) to the set of simultaneous dual integral equations

$F *[\propto M(\xi)+\beta N(\xi), x]=f_{1}(x),|x|<1$

$F *\left[\operatorname{isgn}\{\beta M(\xi)+\alpha N(\xi), x]=f_{2}(x),|x|<1\right.$

$F *\left[i \xi^{-1} M(\xi), x\right]=0|x|<1$

$F *\left[\xi^{-1} N(\xi), x\right]=0|x|<1$

Where

$\alpha=\left(K_{1}-1\right) \Gamma+\left(K_{2}-1\right)$

$\beta=\left(K_{2}+1\right) \Gamma+\left(K_{2}-1\right)$

and the functions $f_{1}(\mathrm{x})$ and $f_{2}(\mathrm{x})$ may be calculated from the prescribed function $\mathrm{p}(\mathrm{x})$ by means of the equations

$$
\begin{aligned}
f_{1}(x)=K_{1}\left(K_{1}+\right. & \Gamma)\left(1+K_{1} \Gamma\right) \mu_{1}^{-1} p(x)-2 K_{1} \Gamma F \\
& *\left[\left(\Gamma+K_{2}\right) \Omega_{1}(\xi)+1(1\right. \\
& \left.\left.+K_{1} \Gamma\right) \Omega_{2}(\xi)\right]
\end{aligned}
$$

$f_{2}(x)=2 K_{1} \Gamma F *\left[i\left\{\Gamma+K_{2}\right) \Omega_{1}(\xi)-\left(K_{1} \Gamma+1\right) \Omega_{2}(\xi)\right]$

It should also be observed that

$\beta^{2}=\propto^{2}=4\left(K_{2}+\Gamma\right)\left(1+K_{2} \Gamma\right) \neq 0$.

\section{Solution of the Simultaneous Dual Integral Equations}

We shall assume that the pressure $p(x)$ is an even function of $\mathrm{x}$. In this case the equations (2.23) and (2.24) reduce to

$$
\begin{aligned}
& F c\left[\propto M(\xi)+\beta N(\xi), x=f_{1}(x), 0<x<1\right. \\
& F s\left[\beta M(\xi)+\beta N(\xi), x=f_{1}(x), 0<x<1\right.
\end{aligned}
$$

$F s\left[\xi^{-1} M(\xi), x=0, x>1\right.$

$F c\left[\xi^{-1} N(\xi), x=0, x>1\right.$

and it is easily shown that

$F c[\alpha M(\xi)+\beta N(\xi), x]=f_{1}(x), 0<x<1$

$F s[\beta M(\xi)+\alpha N(\xi), x]=f_{2}(x), 0<x<1$

$F s[M(\xi), x]=0, x>1$

$F s[N(\xi), x]=0, x>1$

We begin by solving that set simultaneous dual integral equations (3.3)-(3.4), get

$F_{c}[N(\xi), x]=\left|\begin{array}{cc}r,(x) 0<c<1 \\ 0 & x>1\end{array}\right|$

$F_{S}[M(\xi), x]=\left|\begin{array}{c}r_{1},(x) 0<c<1 \\ 0, \quad x>1\end{array}\right|$

As in Lowe grub and Sneddon (1973), it can easily be shown that

$F S[M(\xi), x]=\frac{1}{\pi} \int_{-1}^{1} \frac{r(t) d t}{(x-t)}$

$F_{C}[N(\xi), x]=-\frac{1}{\pi} \int_{-1}^{1} \frac{s(t) d t}{(x-t)}$

Where $r(t)$ and $s(t)$ being even and odd extensions of $r_{1-}(t)$ and $s_{1-}(t)$ respectively to the interval $(1-1,1)$.

With the help of these equations in simultaneous dual integral equations (3.3)-(3.4) reduce to the following singular integral equations. 
$\propto r(x)-\frac{\beta}{\alpha} \int_{-1}^{1} \frac{s(t)}{(x-t)} d t=\widehat{f}_{1}(x),-1<x<1$

$\propto s(x)-\frac{\beta}{\pi} \int_{-1}^{1} \frac{r(t)}{(x-t)} d t=\widehat{f}_{2}(x),-1<x<1$

Where $\widehat{f}_{1}(x)$ and $\widehat{f}_{2}(x)$ are respectively the even and odd extensions of $f_{1}(x)$ and $f_{2}(x)$ to $(-1,1)$.

If we write

$$
\lambda(x)=s(x)+\operatorname{ir}(x) .
$$

Then the above two equations can be written as a single equation

$i \propto(x) \lambda(x)-\frac{\beta}{\pi} \int_{-1}^{1} \frac{\lambda(x) d t}{(x-t)}=g(x),-1<x<1$

Where

$g(x)=\widehat{f}_{1}(x)+i \widehat{f}_{2}(x$. if we write

$\Lambda(\xi)=\frac{1}{2 \pi i} \int_{-1}^{1} \frac{(t) d t}{(t-\rho)}$

and on using Plemelj formula [6]

$\Lambda^{+}(x)-\Lambda^{-}(x)=\lambda(x)$

$\Lambda^{+}(x)-\Lambda^{-}(x)=\frac{1}{\pi i} \int_{-1}^{+1} \frac{\lambda(t)}{(t-x)} d t$.

Then the integral (3.10) reduce to the following Riemann boundary value problem

$\Lambda^{+}(x)+\mu \Lambda^{-}(x)=i(\alpha+\beta)^{-1} g(x),-1<x<1$

Where the constant $\mathrm{K}$ defined by the equation

$$
K=\frac{\beta-\alpha}{\beta+\alpha}=\frac{\Gamma+1}{K_{1}+K_{2}}>0
$$

The solution to the Riemann boundary value problem posed by equation (3.11) is known to be

$(z)=\frac{X(z)}{2 \pi(\alpha+\beta)} \int_{-1}^{1} \frac{q(t) d t}{X^{+}(t)(t-z)}+C X(z)$

where $\mathrm{C}$ is an arbitary complex constant and $\mathrm{X}(\mathrm{z})$ is defined by the equation

$X(z)=(z+1)^{-\frac{1}{2}+i \gamma}(z+1)^{-\frac{1}{2}-i \gamma}$

Where $\gamma=\frac{1}{2 \pi} \log \mu$

In case $g(t)$ is a polynomial, it is known that

$\int_{-1}^{1} \frac{q(t) d t}{X^{+}(t)(t-z)}=\frac{2 \pi i}{1+\mu}\left[\frac{g(z)}{X(z)}-L(z)\right]$ where

$\mathrm{L}(\mathrm{z})=\frac{1}{2 \pi} \lim _{R \rightarrow \infty} \int_{0}^{2 \pi} \frac{g\left(R e^{i Q}\right) R e^{i Q} d Q}{X\left(R e^{i Q}\right)\left(R e^{i Q}-z\right)}$

Hence we have

$\Lambda(z)=\frac{i}{2 \beta}[g(z)-L(x) X(z)+C X(z)]$

Formula for Stress and Displacement Components

From equation (2.9) we deduce immediately that $U_{y}\left(x, 0^{+}\right)-U_{y}\left(x, 0^{-}\right)=F^{*}\left[|\xi|^{-1}\left\{B_{1}-B_{2^{-}} \Omega_{1}-\Omega_{2}\right\}, x\right]$

$=K_{1} \Gamma^{-1} F^{*}\left[|\xi|^{-1} N(\xi), x\right]$

From (2.17), (2.21) on using (2.22), (3.5) and (3.16), it can easily be shown that

$u_{y}\left(x, 0^{+}\right)-U_{y}\left(x, 0^{-}\right)=\frac{1}{K_{1} \Gamma}\left(\frac{2}{\pi}\right)^{1 / 2} \int_{x}^{1} \frac{s_{1}(u) d u}{\left(u^{2}-x^{2}\right)}, 0 \leq x \leq 1$

where gives the difference of the normal component of displacement at the upper and lower surface of the crack. Similarly from (2.8) we have

$u_{x}\left(x, 0^{+}\right)-u_{x}\left(x, 0^{-}\right)=F^{*}\left[i \xi^{-1}\left\{A_{1}-A_{2}+\Omega_{1}-\Omega_{2}\right\}, x\right]$

From equation (2.17) and (2.22) we have

$u_{x}\left(x, 0^{+}\right)-u_{x}\left(x, 0^{-}\right)-\frac{1}{K_{1} \Gamma} \quad\left[i \xi^{-1} M(\xi), x\right]$

From the continuity condition $u_{x}\left(x, 0^{+}\right)=u_{x}\left(x, 0^{-}\right)$, $x<1$, we get the equation

$\int_{0}^{1} r_{l}(x) d x=0$

This condition determines the arbitrary constant in the general solution given by the equation (3.14).

Now from (2.11), (2.12) we get

$\sigma_{y y}\left(x, 0^{+}\right)=-\mu_{1} K_{l}^{-1} F^{*}\left[\left(K_{l}-1\right) A_{l}(\xi)+\left(K_{2}+1\right) B_{l}(\xi), x\right]$

$\left.\sigma_{x y}\left(x, 0^{+}\right)=-\mu_{1} K_{l}^{-1} F^{*}\left[i\left(K_{1}+1\right) A_{l}(\xi)+\left(K_{1}-1\right) B_{1}(\xi)\right\}, x\right]$

Substituting the values of $A_{l}, B_{1}$ from (2.21) and noting that

$\sigma_{y y}\left(x, 0^{+}\right)=-\mu_{1} K_{l}^{-1}\left(K_{2}+\Gamma\right)(1+$

$K_{1} \Gamma F^{*}\left[\alpha M(\xi)+\beta N(\xi)+2 K_{1} \Gamma\left\{\left(\Gamma+K_{2}\right) \Omega_{2}(\xi)\right.\right.$

$\left.\left.+\left(1+K_{l} \Gamma\right) \Omega_{l}(\xi)\right\}, x\right]$

Also as in Lowegrub (1975) the continuity conditions are satisfied if

$F_{s}\left[\xi^{-1} N(\xi), x\right]=0, x>1$

$F_{c}\left[\xi^{-1} M(\xi), x\right]=0, x>1$ 
Substituting the values of $M(\xi)$ and $N(\xi)$ from (2.21) in the above equations and after interchanging the order of integration we have

$\int_{0}^{1} s_{1}(t) d t=0$

$\int_{0}^{1} r_{1}(t) d t=0$

Hence we have

$\int_{0}^{1} \lambda(t) d t=0$

If we consider the physical important case in which the crack is opened by the application of a prescribed constant temperature $T_{0}$ at its surface then

$T(x)=T_{0}(x)=$ constant

and from the equation (2.14) we have

$\psi(t)=\frac{-2 \alpha_{1}\left(1+\eta_{1}\right) T_{0}}{\pi}=T_{1}($ say $)$.

Hence,

$\Omega_{1}(\xi)=T_{1} \sin \xi / \xi^{2}$

We shall also assume that there is no external pressure applied to the surface of the crack, so that $p(x)=0$. Thus we have

$\widehat{f_{1}}(x)=h_{l}\left(\frac{2}{\pi}\right)^{1 / 2} \int_{0}^{\infty} \Omega_{l}(\xi) \sin (\xi x) \cos (\xi t) d \xi d t$

$=h_{1} T_{1}(2 / \pi)^{1 / 2} x$,

and

$\widehat{f}_{2}(x)=-h_{2}\left(\frac{2}{\pi}\right)^{1 / 2}\left[\log \left|1-x^{2}\right|+x \log \left|\frac{1+x}{1-x}\right|+C_{1}\right]$,

where

$h_{1}=2 K_{1} \Gamma\left[\left(\Gamma+K_{2}\right) \alpha_{1}\left(1+\eta_{1}\right)+\left(1+K_{1} \Gamma\right) \alpha_{2}\left(1+\eta_{2}\right)\right] / \alpha_{1}\left(1+\eta_{1}\right)$

and

$h_{2}=2 K_{1} \Gamma\left[\left(\Gamma+K_{2}\right) \alpha_{1}\left(1+\eta_{1}\right)+\left(1+K_{1} \Gamma\right) \alpha_{2}\left(1+\eta_{2}\right)\right] / \alpha_{1}\left(1+\eta_{1}\right)$

Thus

$g(x)=h_{1} T_{1} x(\pi / 2)^{1 / 2}-\frac{i T_{1} h_{2}}{(2 \pi)^{1 / 2}}\left(x^{2}+\frac{x^{2}}{6}\right)+C_{1}$

Now substituting the value of $g(t)$ and $X(t)$ in (3.14) and on using (3.16) we have

$s(x)=-\left(\beta^{2}-\alpha^{2}\right)^{-1 / 2}\left[\left(d_{1} x^{3}+d_{2} x+d_{3}\right) \cos (\omega Q)-\left(C_{1} x^{4}+C_{2} x^{2}\right)+C_{3}\right.$ $\sin (\omega Q)]$. and

$r(x)=-\left(\beta^{2}-\alpha^{2}\right)^{-1 / 2}\left[\left(d_{1} x^{3}+d_{2} x+d_{3}\right) \sin \right.$

$\left.(\omega Q)+\left(C_{1} x^{4}+C_{2} x^{2}+C_{3}\right) \cos (\omega Q)\right]$.

where $d_{1}, d_{2}, c_{1}$ and $d_{3}$ are known constants while $C_{2}$ and $C_{3}$ are unknown to be determined. Since $s(x)$ is even, we have $C_{2}=0$. On using (4.10), we have

$C_{3}=\frac{-\int_{0}^{1}\left[\left(d_{1} x^{3}+d_{2} x\right) \sin (\omega Q)+\left(C_{1} x^{4}+C_{2} x^{2}\right) \cos (w Q)\right] d x}{\int_{0}^{1} \cos (\omega Q) d x}$

\section{RESULTS AND DISCUSSION}

In this paper we have find out displacement components and Thermal Stress in Bonded dissimilar Elastic Half Planes containing a Griffith Crack at the interface.

\section{ACKNOWLEDGEMENT}

I would like to acknowledge the help of received from Dr. P.K. Tripathi Deptt. of Mathematics, D.A.V College Kanpur and Dr. A.P. Dwivedi Ex Prof. Deptt. of Mathematics, H.B.T.U. Kanpur.

\section{REFERENCES}

Brown E.J. and Erdogan F., 1968. Thermal Stresses in Bonded Materials containing cuts on the interface, Int. J. Eng. Sci., 6: 517-529.

Bregman A.M. and Kabsir M.K., 1974. Thermal fracture of bonded dissimilar media containing a penny shaped crack. Int. J. Fracture, 10: 87-98.

England A.H., 1965. A crack between dissimilar media. J. Appl. Mech., 32 (2):400-402.

England A.H., 1971. Complex Variables in Elasticity, Wiley.

Lowengrub M. and Sneddon I.N., 1973. The stress field near a griffith crack at the interface of two bonded dissimilar elastic half-planes. Int. J. Engg. Sci., 11(9):1025-34.

Lowengrub M., 1975. A pair or coplaner cracks at the interface of two bonded dissimilar elastic half planes, Int. J. Engg. Sci., 13:731.

Mushkhelshvile N.I., 1953. Some Basic Problems of the Mathematical theory of Elasticity. P. Noordhof. Ltd., Groningen, The Netherlands. 